Recepción: 20 / 04 / 2017

Aceptación: 20 / 05 / 2017

Publicación: 15 / 07 / 2017
Ciencias de la Educación

Artículo Científico

\title{
El cuento como estrategia didáctica para fomentar los valores del "Buen Vivir"
}

The story as a didactic strategy to promote the values of "Good Living"

\section{El cuento como estratégia didáctica para fomentar os valores do "Buen Vivir"}

\begin{abstract}
María E. Alarcón-Salvatierra ${ }^{\mathrm{I}}$ maria.alarcons@ug.edu.ec

Pablo A. Alarcón Salvatierra II pablo.alarcons@ug.edu.ec

Marjourie E. Ponce-Onofre ${ }^{\text {III }}$ marjourie.ponceo@ug.edu.ec
\end{abstract}

Correspondencia: maria.alarcons@ug.edu.ec 


\section{Resumen}

Este artículo plantea una exposición de los elementos teóricos de la axiología que deben incorporarse en los procesos educativos de la sociedad ecuatoriana, sobre la base del cuento como estrategia didáctica ante la pérdida de valores que se aprecia en la escuela y deriva del proceso de globalización. Para ello, se adoptó la teoría constructivista y sociocrítica como piso teórico para la elaboración de una propuesta factible a ser aplicada en el Centro de Educación Básica "República de el Salvador" de Guayaquil, Ecuador, a los estudiantes del primer año. Buscando con ello brindar opciones a los docentes, que puedan ser llevadas a la práctica educativa y con ello comenzar a revertir el proceso de reversión de valores en los estudiantes, dado que a la larga serán los futuros constructores de la sociedad.

Palabras claves: Diseño; destrezas; formación. 


\section{Abstract}

This article presents an exposition of the theoretical elements of the axiology that must be incorporated in the educational processes of Ecuadorian society, based on the story as a didactic strategy before the loss of values that is appreciated in the school and derives from the process of globalization. For this, the constructivist and sociocritical theory was adopted as theoretical floor for the elaboration of a feasible proposal to be applied to the first year students in the Basic Education Center "República de el Salvador" of Guayaquil, Ecuador. Seeking to offer options to teachers, which can be taken to educational practice and with it begin to reverse the process of reversion of values in students, given that in the long run will be the future builders of society.

Key words: Design; skills; training. 


\section{Resumo}

Este artigo apresenta uma exposição dos elementos teóricos do axiología deve ser incorporada nos processos educacionais da sociedade equatoriana, baseado na história como estratégia de ensino na perda de valores que pode ser visto na escola e deriva do processo de globalização. Para fazer isso, a teoria construtivista e piso teórica sociocrítica para o desenvolvimento de uma proposta viável para ser aplicado no Centro de Educação Básica "República do Salvador" de Guayaquil, Equador, de calouros foi adotada. Buscando proporcionando assim opções de professores que podem ser postas em prática educativa e, assim, começar a reverter a valores de processo de reversão nos alunos, uma vez que acabará por ser os futuros construtores da sociedade.

Palavras-chave: Design; habilidades; treinamento. 


\section{Introducción}

El proceso de globalización va avanzado sostenidamente. Las naciones latinoamericanas y el Ecuador han sido tocados por el efecto homogeneizador de la cultura y la economía occidental. En este nuevo entramado, el país ha sido testigo de la pérdida sostenida de los valores esenciales que distinguían a la sociedad ecuatoriana, al quedar expuesto todo su aparato educativo ante los efectos diluyentes de la transculturación en todos los ámbitos.

Los rasgos identitarios van desapareciendo y con estos, los valores fundamentales que deben regir a las sociedades, incluso hasta distorsionar las funciones esenciales de la escuela.

La búsqueda de alternativas para revertir el proceso de desvaloración social ecuatoriano por parte de los maestros de la escuela, conduce a la adopción del cuento como alternativa didáctica, estratégica e inteligente, dado que permite fomentar, sobre un criterio lúdico constructivo, aquellas prácticas propulsoras de la inventiva y la libertad tan necesarias hoy día en los estudiantes y que a la larga harán posible un ciudadano con capacidad de discernir lo mejor para su entorno y para sí mismo.

Educar en valores más que una opción, es una obligación de los docentes comprometidos con el Buen Vivir como filosofía país y es que la incorporación de esta base axiológica ancestral en todos los ámbitos de la cotidianeidad del país resulta una contrapropuesta de cara a la globalización en curso.

En este contexto, este trabajo contiene una serie de orientaciones y propuestas didácticas para llevar a la práctica valores imprescindibles para la vida en sociedad, bajo una perspectiva que sirva de fundamento a los docentes y padres para una acertada educación.

Está basado en orientaciones y criterios metodológicos precisos, que mediante el diseño y el empleo del cuento dé con una alternativa factible para un trabajo sistemático e intencional, que refuerce los 
preceptos valorativos que se esperan concretar en los ecuatorianos y por lo tanto también en los estudiantes del Ecuador.

Se trata de una vía sencilla pero efectiva, dado el enorme potencial que el cuento tiene sobre los niños y los adolescentes en proceso de formación. Se busca, a través de todo el análisis recogido en estas páginas contribuir en la profundización del debate de una sociedad preocupada por la situación humana que enfrenta, en la que el egoísmo, la perdida de afectividad y la violencia están a la orden del día amenazando con volvernos insensibles, inhumanos ante las necesidades del otro.

Revertir las consecuencias negativas de la globalización es responsabilidad de todos. La familia y la escuela juegan un rol clave en la superación de todos los antivalores que se evidencian en los niños y jóvenes. En este sentido, este artículo no deja de lado esta dualidad, por el contrario, expresa con absoluta determinación que ambos actores (familia y escuela) están obligados a tomar conciencia y a actuar en consecuencia, en aras de lograr una buena educación en los niños y alumnos del Centro de Educación Básica “República de el Salvador”, Guayaquil, en Ecuador.

Para ello, la evaluación de la factibilidad de esta propuesta se realizó considerando a los padres, los docentes y los estudiantes en su conjunto. Tal y como un estudio honesto e interesado en transformar la realidad actual debe asumir.

En este sentido, sirva este trabajo como una propuesta planteada por uno de los actores fundamentales de la sociedad ecuatoriana: los maestros, los docentes, los educadores o profesores, pues como quiera que se le llamen debe tenerse siempre presente la enorme responsabilidad social que en la actualidad y en el futuro cercano tienen para con el futuro del Ecuador y del Buen Vivir. 


\section{Materiales y métodos.}

El marco regulatorio de este artículo está constituido por Buen Vivir y los diversos planes que lo fundamentan como principio filosófico nacional. El problema radica en cómo viabilizar tal práctica y a través de cuáles estrategias.

En tal sentido, el modelo constructivista con un enfoque socio crítico se plantea como pieza clave, ya que pretende la formación de personas como sujetos activos, capaces de tomar decisiones y emitir juicios de valor desde la infancia entrelazando la teoría con la práctica de manera crítica. Pero ello implica la participación activa de profesores y estudiantes que interactúen en el desarrollo de la clase, para reflexionar sobre la comprensión de las estructuras profundas de los elementos axiológicos estructurados en el cuento comparado con su entorno y la sociedad.

Ahora bien, ¿Qué efecto tendría la aplicación de valores a través de los cuentos en el proceso de la enseñanza para fortalecer las relaciones interpersonales en el marco del Buen Vivir de los estudiantes?

A los efectos de dilucidar la hipótesis plantada, este trabajo se desarrolló dentro de los criterios investigativos de la investigación de campo y de tipo explicativa. Igualmente, es una investigación de tipo factible en vista que plantea alternativas de solución expresadas en formatos didácticos tipo cuento, a aplicar por parte de los docentes en el aula de clase.

Desde un punto de vista metodológico, se tiene que el método histórico lógico facilito la comprensión de las actividades escolares acontecidas en periodos anteriores, para desde allí construir un panorama adecuado sobre las estrategias pedagógicas que sirven de fundamento a la práctica de los valores en la escuela. Posteriormente, el método deductivo viabilizó la comprensión 
general del problema objeto de estudio mediante la aplicación de un instrumento tipo encuesta, cerrada, de tipo probabilístico, a una muestra de 17 docentes, 26 estudiantes y 140 representantes.

Estas mediciones y consultas arrojaron resultados reveladores en lo que respecta a los acuerdos generales sobre la necesidad de educar en valores, prácticamente existe un consenso general sobre la utilización del cuento como instrumento pedagógico. No obstante, quedan algunos elementos a considerar en el futuro cercano si realmente este planteamiento, este estudio e indagación, será considerado como base para revertir la pérdida de valores esenciales en los estudiantes de primer año de la escuela "República de El Salvador".

\section{Resultados.}

En tocante a los profesores tenemos que existe una opinión consensuada sobre el empleo del cuento como herramienta didáctica; sin embargo, una porción mayoritaria de los docentes, es decir, el $82 \%$ no ha adecuado el espacio en el que imparte la enseñanza para la ubicación de cuentos o libros. Destaca en este caso la subvaloración sobre el papel de mediación que cumple el espacio, el entorno escolar, en el proceso de formación del estudiante.

Por lo tanto, inicialmente, la relación recíproca entre el espacio de clase y el alumno no es considerada un elemento prioritario por parte del maestro a la hora de implementar la estrategia didáctica. Estos resultados demuestran que los métodos empleados son tradicionales: profesor impartiendo conocimientos al alumno. De allí la pertinencia del método constructivista en el que el maestro se involucra, practica, indaga y construye juicios teóricos, morales y éticos sobre el entorno que rodea al estudiante en tanto mecanismo para afianzar valores. 
Aunque es un porcentaje minoritario, destaca el hecho de que un $12 \%$ de los docentes no considere necesario contribuir con la formación moral en los estudiantes., dejando en entredicho su responsabilidad al no considerar que la educación es un proceso dual que relaciona permanentemente a la familia y a la escuela. No obstante, un $88 \%$ de los profesores considera el fomento de los valores morales una obligación. Todo esto da cuenta de la claridad de su rol social y humano en el proceso enseñanza aprendizaje.

Dentro de este contexto de aprobación general, destaca el hecho de que el docente (un 76\%) comprende y conoce la filosofía del Buen Vivir establecida como proyecto nacional dentro de la Constitución Nacional del Ecuador y el marco de desarrollo educativo existente: el plan decenal. Pese a ello no puede obviarse que un $24 \%$ desconoce el contenido de la reforma curricular establecida en al Plan Decenal por la Educación, es decir, ya el patrón referencial desde el cual impulsar un planteamiento didáctico en torno al cuento comienza a ser más confuso. Esto sin duda conlleva las siguientes repercusiones que veremos más adelante.

Ahora bien, respecto a los resultados arrojados para el caso de los representantes se tiene que la mayoría, el $97 \%$ acepta de buena manera que el docente diseñe un cuento junto al estudiante a objeto de fomentar los valores. Con ello se puede observar un consenso prácticamente absoluto entre docentes y representantes sobre el empleo del cuento en el aula.

Ahora bien, cuando se traslada la responsabilidad de leer, de fomentar la práctica de la lectura, los resultados demuestran que los padres no tienen un espació acondicionado para ello en el hogar: un $51 \%$ para ser precisos. Esto demuestra que, pese a existir consenso sobre la necesidad del cuento, los padres trasladan en su mayoría, la responsabilidad a los maestros de escuela. 
Dentro de todo este panorama positivo, emite señales de alerta un factor importantísimo y es que cuando se intenta saber sí se conocen las intenciones del Estado ecuatoriano en materia educativa (a través de sus planes, currículos y leyes), la inmensa mayoría no lo hace. Es decir, un $84 \%$ de los entrevistados. Este factor resulta relevante, dado que hay un enorme esfuerzo nacional por establecer una relación de responsabilidad a nivel educacional con la sociedad que a todas luces luce infructuoso.

En esta sección aparece uno de los hallazgos más preocupantes. La percepción sobre la necesidad de la práctica de valores en los estudiantes no es del todo concluyente en un sentido positivo. Solo el $44 \%$ de los alumnos reconoce estar inmerso en un sistema de normas de convivencia grupal. Hay vacilaciones respecto al apego a un sistema de valores en la escuela. ¿Qué quedará en el futuro para la sociedad?

Si se infieren las razones de estos resultados, se puede apreciar que la buena intención de los padres no se traduce en un esfuerzo a lo interno de la familia que consiga reforzar la práctica de valores para la vida, para la escuela.

Al plantear el problema valorativo desde la perspectiva de la libertad y de la expresión emocional de sentimientos, se llegan a cifras que preocupan mucho. Sólo el $47 \%$ de los estudiantes se expresa de manera espontánea. Si sumamos a los que no lo hacen, el ítem que lo hace a veces, se llega a un $53 \%$ de la muestra, lo que indica inconsistencia en la expresión de las emociones, quizás influenciado porque en el caso de reconocerse con un persona con derechos y responsabilidades los resultados son similares.

De todo ello se desprende, el que sea natural, como lo demuestra el estudio que únicamente el $31 \%$ respete las diferencias individuales y de grupos. El individualismo en la sociedad fija aquí los 
cánones de comportamiento estudiantil ante su comunidad y entorno. De hecho, esta sección revela de manera inequívoca que estamos ante una crisis de valores como la solidaridad, el respeto mutuo y la colaboración, en etapas de formación educativa prácticamente iniciales y para acotar, que pueden ser resueltas aplicando estrategias didácticas renovadas y cónsonas con la nueva realidad país.

Para fortalecer esta opinión, las debilidades del estudiante ante la lectura, su capacidad de comprensión y coherencia discursiva están a niveles igualmente bajos. Con ello, puede observarse, y más aún comprenderse la enorme debilidad que existe hoy en la sociedad ecuatoriana que se forma en el aula de clase con vistas a los roles que desempeñará mañana. Profesores y padres, escuela, familia y estado deben comprometerse más con un futuro mejor sobre la base del componente fundamental de toda sociedad: el hombre en formación.

De estos datos se pueden desprender ciertas observaciones de utilidad para todo aquél que desee cooperar en el enorme reto educativo que plantea el presente. Esperemos que lo positivo y lo negativo alerten y alienten es este sentido.

\section{Discusión.}

El agotamiento dela concepción tradicional de enseñanza aprendizaje, que se ejerce desde la unilateralidad comienza a dar muestras de agotamiento. Los resultados ulteriores que se buscan en el proceso educativo: un ser humano apto para vivir en sociedad, parecieran ser contradictorios a partir del análisis de la perspectiva de los estudiantes entrevistados.

¿es sólo responsabilidad del docente alcanzar este objetivo? O ¿los padres también tiene su cuota de responsabilidad? 
Estas preguntas deben responderse evaluando la incidencia real del Estado en los resultados a largo plazo de la política educativa y de la escuela como espacio acorde para impulsar estrategias novedosas frente al proceso de globalización. Pareciera, que por más esfuerzos que la familia haga en conjunto con los educadores, todo acaba siendo de una incidencia moderada si no se comprende la relevancia de impulsar un papel más activo y envolvente de la institucionalidad como un todo. Porque a fin de cuentas ¿Qué representan es el Estado, la familia y la escuela? Si no instituciones formales y simbólicas para el ser humano.

Pese a que la adopción del cuento en su mayoría cuenta con un apoyo increíble, requiere también que la lectura, es decir de un aspecto de fondo, con la intención de que sea asumida como un aspecto trascendental en todos los espacios de la cotidianeidad, tanto por los padres como por los hijos y los docentes. En el cultivo del placer de leer, de construir y de todas las maneras posibles un mundo distinto, así sea imaginario, subyace la salida más razonable a la grave crisis de valores que padecemos en todos los ámbitos del Ecuador

Por lo tanto, ¿No sería pertinente ampliar la ecuación explicativa sobre la pérdida de valores e incorporar el papel, activo o no, que juega el aparato de Estado en los resultados actuales? Se asume que sí, que es pertinente.

Mientras tanto este articulo permite ahondar más en el asunto que verdaderamente interesa, a saber: fomentar los valores del Buen Vivir a través del empleo del cuento en las aulas de clase.

\section{Recomendaciones.}

Inculcar la práctica de una verdadera convivencia a partir del respeto hacia los demás, durante todos los niveles de educación y de manera continua. 
Es necesario retomar y evaluar críticamente los aciertos de cada uno de los modelos que han existido en el proceso enseñanza- aprendizaje. Su estudio permitirá implementar la mejor estrategia para evolucionar hacia nuevas formas y herramientas de enseñar a los alumnos.

\section{Conclusiones}

Después de haber analizado e interpretado los resultados de las encuestas realizadas a los docentes y representantes y la lista de cotejo realizada a los estudiantes de Primer Año de Educación General Básica de la institución "República de el Salvador", los resultados nos confirman la falta de valores en casa y en la escuela y el desconocimiento de estrategias didácticas por medio de cuentos que favorecen al fortalecimiento de valores y el fomento del Buen Vivir.

En los nuevos escenarios, por los cuales estamos transitando, se puede apreciar que a pesar de la globalización y el permanente cambio del contexto existe la apreciación de los valores a nivel de educación.

Las dificultades que presentan los estudiantes al momento de integrarse a diferentes actividades dentro de la institución y hacer partícipe a la familia revela la necesidad de seres críticos y creativos cultivados desde mecanismos más asertivos e interactivos.

Se ratifica la importancia de presentar cuentos diseñados por maestros y estudiantes en aras de fortalecer valores y fomentar el Buen Vivir dentro de la comunidad educativa objeto de estudio.

\section{Bibliografía}

Acosta Farrietta Édgar. (2002). El Libro De Los Valores, El Universo, Casa Editorial El Tiempo, Guayaquil Ecuador.

Ander - Egg Ezequiel (2000),.Métodos y Técnicas de investigación Social III, Cómo Organizar el Trabajo de Investigación, Editorial Lumen, Buenos Aires México.

Armstrong Thomas (2000), Inteligencia Múltiples en el Aula, Guía Práctica Para Educadores Prólogo De Gardner. Editorial Paidós. Barcelona/Buenos Aires/México. 
Asamblea Nacional Constituyente Ley Orgánica De Educación Intercultural (2011), Registro Oficial No 417. Editorial EDUQUIL, Universidad de Guayaquil, Guayaquil - Ecuador.

Bixio Cecilia, (2005). Como Planificar y Evaluar en el Aula Propuestas Y Ejemplos. Editorial Paidós Educadores. Argentina.

Blinder, (2004). Clínica Psicoanalítica con Niños. Editorial Síntesis, Madrid.

Boan Susana (2007), Escuela para Maestros, Enciclopedia de Pedagogía Práctica. Editorial By cadiex, International, S.A. Montevideo - Uruguay.

Carreño Manuel Antonio, (2012). Manual de Urbanidad y Buenas Costumbres Editorial RADMANDÍ, Ecuador - Quito.

Código de Convivencia (2010) del Centro de Educación Básica “República de El Salvador”, Guayaquil Ecuador.

Constitución De La República Del Ecuador (2008). Publicado en el Registro Oficial, Editorial EDUQUIL Universidad de Guayaquil, Quito -Ecuador.

Declaración Final De La XX Cumbre Iberoamericana. (Declaración De Mar del Plata), Argentina (2010), bajo el tema "Educación Para La Inclusión Social". Editorial.

Domenech Yial Ana Lorena Cecilia Amaluisa Fiallos. (2010). Como Trabajar El Buen Vivir En El Contexto Educativo, es una obra colectiva creada y diseñada por el departamento de Ediciones Educativas de Santillana S.A. bajo la Dirección Editorial de Ana Lucia de Escobar. Ecuador.

Falieres Nancy y Antolín Marcela. (2006), Como Mejorar el aprendizaje en el aula y poder evaluarlo. Editorial Grupo CLASA, Buenos Aires.

García Batista Gilberto Dr. Nocedo de león Irma M.Sc. (2009). Metodología de la investigación, Primera Parte, Editorial Pueblo y Educación, La Habana - Cuba.

García Batista Gilberto Dr. Nocedo de león Irma M.Sc. (2009). Metodología de la investigación, Segunda Parte, Editorial Pueblo y Educación, La Habana - Cuba.

García Joaquín, (2010). Educando Con Valores, Virtudes, Ejemplos y Más. Ediciones MIRBET S.A.C, Perú. Garza Rosa María y Leventhal Susana (2003), Aprender cómo aprender Tercera Edición, Euroméxico, S.A de C.V. México.

Grados L. Fernando, (2006), El Tesoro De Los Valores Más De Cien Conceptos, Reflexiones, Testimonios y Moralejas. Editorial ARBOLEDA. Perú.

Grass Pedrals, Juan, (2003). La educación de valores y virtudes en la escuela. Teoría y práctica. Editorial D. F. Trillas, México.

Hernández-Fernández y Baptista. (2010). Metodologías de la investigación. Quinta Edición. Mc Graw Hill. 
Kohlberg, Lawrence. (1981), The philosophy of moral development. Moral stages and the idea of justice. San Francisco, CA: Harper \& Row Pubs.

Latapí Sarre Pablo, (2004). "La laicidad escolar: cinco vertientes para su investigación” en Diálogo y debate de cultura política.

López Reyes Alex, (2006). Inteligencia múltiple, cómo descubrirla y desarrollarla Editorial ARBOLEDA. Lima - Perú.

Mejía Sandoval, (2006). Representación de la lectura, el libro y las bibliotecas en la literatura infantil. Tesis, Licenciatura en Bibliotecología, Universidad Nacional Autónoma de México, Facultad De Filosofía Y Letras.

Ministerio de Bienestar Social, Código de la niñez y adolescencia (2003), Publicado Por Ley No. 100. En Registro Oficial 737, Ecuador.

Ministerio De Educación Del Ecuador, Actualización y Fortalecimiento Curricular de La Educación General Básica (2010). Ecuador.

Ministerio de Educación y Cultura, códigos de convivencia, acuerdo ministerial $\mathrm{N}^{\circ} 1962$ del 18 de julio de 2003.

Moreno, (2000). Análisis de contenidos de los papeles, estereotipos y normas de géneros en los cuentos de hadas. Tesis Inédita de la licenciatura en Psicología de La Universidad Nacional Autónoma de México. México.

Morín Edgar (2010). "La Cabeza Bien Puesta”, Editorial EDUQUIL Universidad de Guayaquil, Guayaquil Ecuador.

Organización para la cooperación y el desarrollo. (2002). Conocimientos y actitudes para la vida. Resultados de PISA 2000. Ciudad de México, México: Aula Santillana XXI.

Ostrovsky Graciela, (2006). Como construir competencias en los niños y desarrollar su talento: para padres y educadores, $1^{\text {a }}$ ed. Editorial Grupo CLASA, Buenos Aires- Argentina.

Pacheco Gil Oswaldo, (2004). Teoría y práctica de proyectos educativos, Editorial PAESCA, Guayaquil Ecuador.

Palos Rodríguez, J. (2000). Educación y desarrollo sostenible. Monografías de OEI.

Papalia, (2001). Desarrollo Humano. Editorial Mac Graw-Hill, Colombia.

Pavez Guzmán María Mercedes, Coloma Tirapegui Carmen y Maggiolo Landaeta Mariangela, (2008), El desarrollo narrativo en niños una propuesta práctica para la evaluación y la intervención en niños con trastornos de lenguaje. Por Barcelona - España.

Plan Nacional Decenal de Educación del Ecuador (2006-2016). Ecuador.

República del Ecuador Plan Nacional de Desarrollo, (2009 - 2013), Plan Nacional para el Buen Vivir. Disponible en www.educacion.gob.com.ec

Rugarcía Torres, Armando, (2004). Los valores y las valoraciones en la educación, Editorial Trillas. México. 
Ruiz Corbella, Marta, (2003). Educación Moral: Aprender a ser, aprender a convivir, Editorial Ariel Educación, Barcelona.

Sánchez Isidro y Vidal José A. (2010), Escuela de actividades y valores, Editorial Océano, Barcelona España.

Schneider Sandra (2004). Como Desarrollar La Inteligencia Y Promover Capacidades, Circulo Latino Austral S.A. Buenos Aires - Argentina.

Sophie, Bolo Libros del Rincón, (2005). Vivir Con Los Demás, Editorial ULTRA, México.

Soto P. Rogelio, (2006), La Educación Basada en Valores, Editorial Palomino E.I.R.L, Perú - Lima.

Unell Barbara C. y Vyckoff Jerry L. (2004). 20 Valores Que Puede Transferir A Sus Hijos, Editorial ARBOLEDA. Lima - Perú.

UNESCO Y Living Values: an Educational Program, (2006). Taller internacional integración de valores en los programas y servicios de la primera infancia. Paris.

Vásquez Valerio Francisco Xavier, (2006), Modernas Estrategias Para La Enseñanzas, Tomo 1 y 2 Ediciones Euroméxico, S.A de C.V. México. 